\title{
Rearfoot and Tibial Motion during Gait Associated with Medial Tibial Stress Syndrome: A Systematic Review
}

\author{
Hyung-Pil Jun ${ }^{1}$ PhD, Eunwook Chang ${ }^{2}$ PhD \\ ${ }^{1}$ Department of Physical Education, Dong-A University, Busan; ${ }^{2}$ Department of Kinesiology, Inha University, Incheon, Korea
}

PURPOSE: The purpose of this study was to confirm the kinematic characteristics of overuse leg injuries during gait through a systematic review.

METHODS: Electronic databases from 1960 to 2018 were used to search studies. Physiotherapy Evidence Database Scale was used for quality of studies. Effect sizes (ES) of between-groups and its $95 \%$ confidence intervals were calculated.

RESULTS: Seven studies were selected for review. The average Physiotherapy Evidence Database Scale score was 4.3 of 10 -point scale. The ES for maximum rearfoot eversion from 5 studies ranged from -0.34 to 0.67 with 3 of 5 studies statistically significant. The ES of eversion excursion from 4 studies ranged from -0.24 to 2.37 and 3 of 4 studies showed statistically significant. The ES of maximum eversion velocity ranged from 0.29 to 0.38 from all of 2 studies. With abduction, the ES for abduction excursion ranged from -0.04 to 0.35 , and 2 of 3 studies revealed statistically significant. The ES of maximum abduction velocity showed statistically significant with the ES of 0.23 and 0.61 . However, maximum abduction from 3 studies showed non-significant with ES ranges from 0.00 to 0.62 .

CONCLUSIONS: The rearfoot motion (eversion) associates with overuse leg injuries during gait more than tibial motion (internal rotation).

Key words: Medial tibial stress syndrome, Kinematics, Gait, Injury risk

\section{INTRODUCTION}

Medial tibial stress syndrome (MTSS), a term that related to exercise related leg pain, shin splints, or stress fractures, is one of the most common overuse lower leg injuries among runners and military personnel [1]. Previous studies have reported that MTSS accounts for 6 tol5 percent of all running related injuries [2,3]. In addition, a high incidence of this injury was observed in military recruits of whom up to 35 percent developed MTSS [1]. This condition is characterized by a pain along the distal posterior-medial aspect of the tibia [4].

Due to the high incidence of this injury, many prospective and retrospective studies [5-12] examined many suspected kinematic risk factors for overuse leg injuries during gait cycle. However, the findings from these previous studies are controversial results. Even though these previ- ous literatures examined kinematic variables including ankle, knee, and hip joint, some studies $[1,10,13-15]$ found the significant relationship between lower extremity alignment (e.g., foot posture; pronation) and overuse leg injuries. Based on these findings, this review paper focused on ankle joint kinematic variables to confirm whether there is a relationship between pronation and overuse leg injuries during gait or not, since a relationship between alignment and overuse leg injuries was found.

Numerous studies $[8,9,11,12]$ found that kinematic variables of rearfoot and tibial motion such as peak rearfoot eversion, maximal foot abduction, eversion/abduction excursion, maximal eversion/abduction velocity in overuse leg injury group were greater than the healthy leg group. On the other hand, similar studies [5-7] identified that there was no significant difference on kinematic variables of rearfoot and tibial motion between overuse leg injury and healthy group. As reported

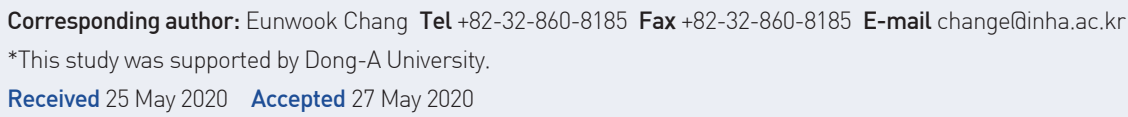


above, the kinematic risk factors of overuse leg injuries exist in the previous literatures but inconsistent and conflicting results were found. $\backslash$ However, kinematic variables of rearfoot and tibial motion from previous studies are related with the deviation of the subtalar joint axis that influences subtalar joint motion in the frontal and transverse planes. The subtalar joint axis is deviated about $41^{\circ}$ to $42^{\circ}$ from transverse plane [16]. This normal deviation produces the pronation of rearfoot is a triplanar motion in the closed kinetic chain and is a combination of adduction and plantar flexion of the talus and eversion of the calcaneus, which causes internal rotation of the tibia [17]. Excessive pronation can increase tibialis posterior tension at the periosteal interface that results in leg overuse injuries such as shin splints, MTSS, and stress fractures [18].

Thus, the purpose of this paper was to determine if rearfoot and tibial motion are associated with overuse injuries during gait. It was hypothesized that individuals with overuse injuries might have a more subtalar joint motion in the frontal and transverse planes. The finding of this systematic review study will confirm the risk factors associated with MTSS during gait and enable the clinicians to develop evidence-based prevention and rehabilitation programs for runners.

\section{METHODS}

\section{Search strategy}

In order to include all potential studies, we used broad terms to search relevant studies. The broad terms, used for this review are different classifications of the overuse leg injuries. All relevant studies were searched through PubMed, CINAHL plus, and SPORTDiscus. The search was limited to human subjects and articles published in the English language between 1960 and 2018 using the search key words medial tibial stress syndrome; exercise related leg pain; shin splints; tibial stress fracture; combination of all the above terms. In order to identify and include other potential studies, all retrieved studies were cross-referenced as a final step for study selection. Fig. 1 illustrates the steps of search terms.

\section{Study selection}

A reviewer $(\mathrm{HJ})$ performed the review process on all retrieved studies. Each individual study was identified and selected based on the criteria. The criteria for study inclusion were used as follows:

1) Studies had to focus on medial tibial stress syndrome/exercise related leg pain/shin splints/tibial stress fracture;

2) Studies had to evaluate kinematic variables related to rearfoot angle and/or tibial rotation;

3) Studies had to include the necessary statistical values

However, meta-analysis and review papers were excluded through our search procedure. Also, oral presentations and free communication were excluded. A reviewer did not perform contact with the authors for this paper.

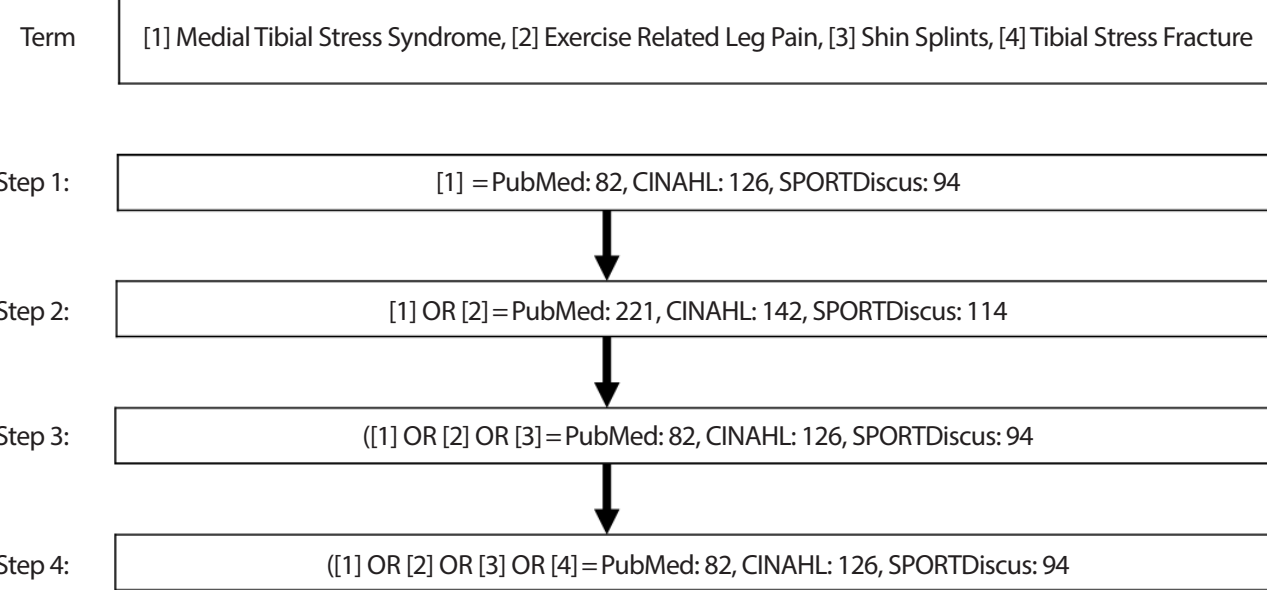

Final search term Medial Tibial Stress Syndrome OR Exercise Related Leg Pain OR Shin Splints

Fig. 1. The progress of search terms. 
In addition, cross reference was performed on all selected studies. A total of seven studies were eligible for inclusion [5-9,11,12]. These studies were rated using the Physiotherapy Evidence Database (PEDro) which is an objective method with 10-point scale to determine the quality of methodology in each study. The details of the literature search and study selection were shown in Fig. 2.

\section{Data extraction and analysis}

Table 1 shows details of the allocation and blinding in all seven studies. We extracted mean and standard deviation of the rearfoot and tibia kinematic variables from both injured and control groups in each retrieved study to calculate Cohen's $d$ effect size $\left(d=\frac{\overline{\mathrm{X}}_{1}-\overline{\mathrm{X}}_{2}}{\mathrm{~s}}\right)$ and confidence interval values (reported in Table 3 ) to show the strength of the relationship. The calculated effect size and its confidence interval values were used to make graphs in Fig. 3 through 8. Even though they have different names of outcome variables depend on 2-dimensional (D) or 3-D analysis, we categorized them in each figure by representing same

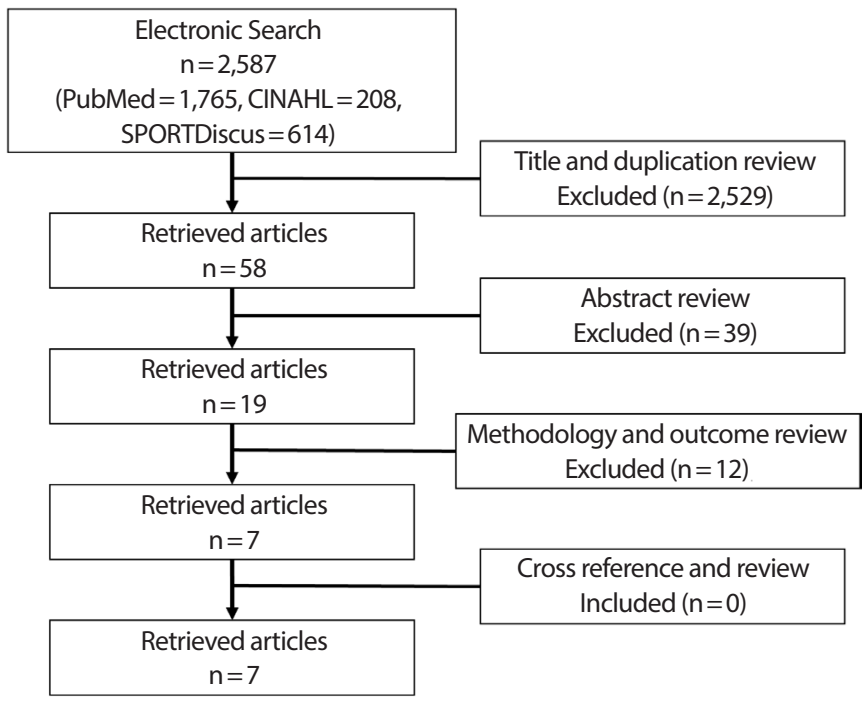

Fig. 2. Initial literature search and selection of studies for inclusion in systematic review. outcome variable. The variables measured in 2-D includes total rearfoot movement [7], while the other studies measured maximum eversion, eversion excursion, maximum eversion velocity, maximum foot abduction, foot abduction excursion, maximum foot abduction velocity [11, 12], Achilles tendon angle at touchdown, total change in Achilles tendon angle [6], peak tibial internal rotation, peak rearfoot eversion [8], rearfoot eversion [9], peak foot abduction, and foot abduction excursion [5] in the 3-D.

\section{RESULTS}

A total of 7 studies were identified and selected in this review. The databases (PubMed, CINAHL plus, and SPORTDiscus) provided a total of 2,587 articles with duplication across three databases. 2,529 of those articles were excluded because those did not meet the criteria clearly through the title and abstract review.

After the review process, specific information was extracted from each selected study on: (1) type of study design; (2) inclusion and exclusion criteria; (3) outcome measure. Only two studies were conducted with prospective cohort study design while five studies used cross-sectional study design. None of selected studies were concealed the allocation and blinded as summarized in Table 1. Table 2 provides a brief

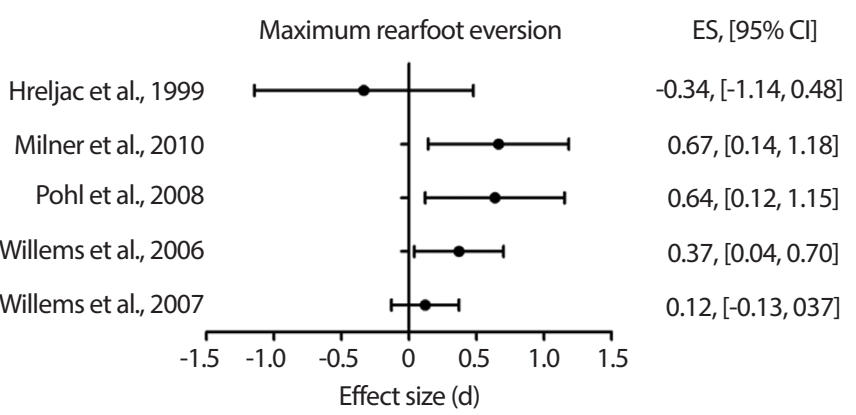

Fig. 3. Maximum rearfoot eversion Cohen's d effect size and 95\% confidential interval.

Table 1. Allocation conceal and blinding

\begin{tabular}{|c|c|c|c|c|c|}
\hline Conceal and Blinding & $\begin{array}{l}\text { Random } \\
\text { allocation }\end{array}$ & $\begin{array}{c}\text { Allocation was } \\
\text { concealed }\end{array}$ & $\begin{array}{c}\text { Blinding of all } \\
\text { subjects }\end{array}$ & $\begin{array}{l}\text { Blinding of all } \\
\text { therapists }\end{array}$ & $\begin{array}{c}\text { Blinding of all } \\
\text { assessors }\end{array}$ \\
\hline Franettovich et al. (2010) & $\mathrm{N} / \mathrm{A}$ & $\mathrm{N} / \mathrm{A}$ & $\mathrm{N} / \mathrm{A}$ & $\mathrm{N} / \mathrm{A}$ & $\mathrm{N} / \mathrm{A}$ \\
\hline Hreljac et al. (1999) & $\mathrm{N} / \mathrm{A}$ & $\mathrm{N} / \mathrm{A}$ & $\mathrm{N} / \mathrm{A}$ & N/A & N/A \\
\hline Messier et al. (1988) & $\mathrm{N} / \mathrm{A}$ & $\mathrm{N} / \mathrm{A}$ & $\mathrm{N} / \mathrm{A}$ & $\mathrm{N} / \mathrm{A}$ & $\mathrm{N} / \mathrm{A}$ \\
\hline Milner et al. (2010) & $\mathrm{N} / \mathrm{A}$ & $\mathrm{N} / \mathrm{A}$ & $\mathrm{N} / \mathrm{A}$ & $\mathrm{N} / \mathrm{A}$ & $\mathrm{N} / \mathrm{A}$ \\
\hline Pohl et al. (2008) & $\mathrm{N} / \mathrm{A}$ & $\mathrm{N} / \mathrm{A}$ & $\mathrm{N} / \mathrm{A}$ & $\mathrm{N} / \mathrm{A}$ & $\mathrm{N} / \mathrm{A}$ \\
\hline Willems et al. (2006) & $\mathrm{N} / \mathrm{A}$ & $\mathrm{N} / \mathrm{A}$ & $\mathrm{N} / \mathrm{A}$ & $\mathrm{N} / \mathrm{A}$ & $\mathrm{N} / \mathrm{A}$ \\
\hline Willems et al. (2007) & N/A & N/A & N/A & N/A & N/A \\
\hline
\end{tabular}


Table 2. Study inclusion criteria, number of subjects, and outcome measures

\begin{tabular}{|c|c|c|c|c|c|}
\hline Author & Design & Inclusion/exclusion criteria & Subjects & Type of outcome measure & PEDro \\
\hline Willems et al. (2006) & $\begin{array}{l}\text { Prospective } \\
\text { cohort }\end{array}$ & $\begin{array}{l}\text { Inclusion: Physical education students } \\
\text { Exclusion: A history of a surgical procedure involving the } \\
\text { lower leg, ankle or foot, A history of an injury to the lower } \\
\text { leg, ankle or foot within } 6 \text { months before the start of the } \\
\text { study. }\end{array}$ & 400 & $\begin{array}{l}\text { Maximal eversion position }\left({ }^{\circ}\right) \\
\text { Excursion eversion }\left({ }^{\circ}\right) \\
\text { Maximal eversion velocity }\left({ }^{\circ} / \mathrm{s}\right) \\
\text { Excursion abduction }\left({ }^{\circ}\right) \\
\text { Maximal abduction velocity }\left({ }^{\circ} / \mathrm{s}\right)\end{array}$ & 4 \\
\hline Willems et al. (2007) & $\begin{array}{l}\text { Prospective } \\
\text { cohort }\end{array}$ & $\begin{array}{l}\text { Inclusion: Physical education students. } \\
\text { Exclusion: A history of a surgical procedure involving the } \\
\text { lower leg, ankle, or foot, or a history of an injury to the low- } \\
\text { er leg, ankle, or foot within } 6 \text { months before }\end{array}$ & 400 & $\begin{array}{l}\text { Maximal eversion position }\left({ }^{\circ}\right) \\
\text { Excursion eversion }\left(^{\circ}\right) \\
\text { Maximal eversion velocity }\left({ }^{\circ} / \mathrm{s}\right) \\
\text { Maximal abduction position }\left({ }^{\circ}\right) \\
\text { Excursion abduction }\left(^{\circ}\right) \\
\text { Maximal abduction velocity }\left({ }^{\circ} / \mathrm{s}\right)\end{array}$ & 4 \\
\hline Messier et al. (1988) & Cross-sectional & Inclusion: Runners who had been running a minimum 10 & 36 & Total rearfoot movement $\left(^{\circ}\right)$ & 4 \\
\hline
\end{tabular}
miles/wk for at least 1 year. (Control: runners who had no history of overuse injuries, Shin splints: runners diagnosed as having shin splints)

Exclusion: N/A

Hreljac et al. (1999) Cross-sectional Inclusion: Runners had been running on a regular basis for a minimum of 3 years (Injury-free: runners who had never sustained an overuse injury throughout their running careers, Injured: runners who had suffered at least one overuse injury that could be attributed to running.)

Exclusion: Runners who had been injured at or below knee, current pain, and had not returned to training regularly for a t least 3 months after recent injury.

Milner et al. (2010) Cross-sectional Inclusion: Femlae distance runners between the ages of 18 and 45 years, who reported running at least $32 \mathrm{~km} / \mathrm{wk}$ (Stress fracture: a history of tibial stress fracture, Control: no previous lower extremity bony injuries).

Exclusion: A current injury, a history of cardiovascular pathology, abnormal menses (missed more than 3 consecutive monthly periods in the previous 12 months), and pregnant or suspected they were pregnant.

Franettovich et al. Cross-sectional Inclusion: Female (ERLP: a history of ERLP within the 12 (2010) months before the study, Control: no current lower limb injury or a history of lower limb injury in the past 12 months that required treatment or interfered with work or leisure.) Exclusion: A history of surgery to the lower limb, had blood clotting or bleeding abnormalities, or had a neurologic or a cardiac condition. A medical diagnosis of compartment syndrome or tibial stress fracture within the last 12 months.

Pohl et al. (2008) Cross-sectional Inclusion: Female runners between the ages of 18 and 45 years and rearfoot strikers (Stress Fracture: a previous TSF which had been confirmed by a medical doctor, Control: no previous lower extremity bony injuries)

Exclusion: current injury (or still recovering from injury), a history of cardiovascular pathology, abnormal menses (missed more than three consecutive monthly menstrual cycles in the last 12 months), and pregnant or suspected pregnant.

24 Achilles tendon angle at touchdown $\left(^{\circ}\right)$

Total change in Achilles tendon angle $\left(^{\circ}\right)$

60

Peak abduction $\left({ }^{\circ}\right)$

Peak rearfoot eversion $\left(^{\circ}\right)$
5

4

28 Abduction $\left({ }^{\circ}\right)$

Total abduction excursion $\left({ }^{\circ}\right)$

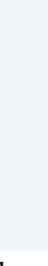

60 Rearfoot eversion $\left(^{\circ}\right)$

4

summary of the specific inclusion and exclusion criteria, and the methodology for the individual studies in this review. Also, the specific kinematic variables related to rearfoot eversion and tibial internal rotation were reported in Table 2. To assess the quality of selected studies, Physiotherapy Evidence Data-base (PEDro) score of each individual study were reported in Table 2. A mean of PEDro score is 4.3 out of 10-point scale.

A total of 1,008 individuals were participated in all selected studies. The main inclusion criteria of subjects is adults between 18 and 45 years old. Two studies $[11,12]$ included collegiate healthy population because 
Table 3. Maximum rearfoot eversion

\begin{tabular}{lcccccccc}
\hline \multirow{2}{*}{ Authors } & \multicolumn{3}{c}{ Treatment } & & \multicolumn{3}{c}{ Control } \\
\cline { 2 - 4 } \cline { 7 - 9 } & $\mathrm{n}$ & Mean $\left(^{\circ}\right)$ & SD & & $\mathrm{n}$ & Mean $\left(^{\circ}\right)$ & SD \\
\hline Hreljac et al., 1999 & 12 & 4.0 & 4.1 & & 12 & 5.9 & 6.7 \\
Milner et al., 2010 & 30 & 11.7 & 4.2 & & 30 & 9.0 & 3.9 \\
Pohl et al., 2008 & 30 & 11.5 & 4.3 & & 30 & 8.8 & 4.1 \\
Willems et al., 2006 & 46 & 9.6 & 5.81 & & 167 & 7.66 & 5.05 \\
Willems et al., 2007 & 75 & 10.48 & 3.82 & & 334 & 10.03 & 3.8 \\
\hline
\end{tabular}

Table 4. Eversion excursion

\begin{tabular}{lllllllll}
\hline \multirow{2}{*}{ Authors } & \multicolumn{3}{c}{ Treatment } & & \multicolumn{3}{c}{ Control } \\
\cline { 2 - 4 } \cline { 8 - 9 } & $\mathrm{n}$ & Mean $\left(^{\circ}\right)$ & $\mathrm{SD}$ & & $\mathrm{n}$ & Mean $\left(^{\circ}\right)$ & $\mathrm{SD}$ \\
\hline Hreljac et al., 1999 & 11.7 & 12 & 4.1 & & 12.8 & 12 & 5.1 \\
Messier et al., 1988 & 16.72 & 17 & 1.36 & & 13.5 & 19 & 1.36 \\
Willems et al., 2006 & 15.47 & 46 & 5.46 & & 13.81 & 167 & 4.39 \\
Willems et al., 2007 & 19.5 & 75 & 4.91 & & 18.06 & 334 & 4.53 \\
\hline
\end{tabular}

Table 5. Maximum eversion velocity

\begin{tabular}{lcccccccc}
\hline & \multicolumn{3}{c}{ Treatment } & & \multicolumn{3}{c}{ Control } \\
\cline { 2 - 4 } \cline { 8 - 9 } Authors & $\mathrm{n}$ & $\begin{array}{c}\text { Mean } \\
(\% / \mathrm{s})\end{array}$ & SD & & $\mathrm{n}$ & $\begin{array}{c}\text { Mean } \\
(\% / \mathrm{s})\end{array}$ & SD \\
\hline Willems et al., 2006 & 440.73 & 46 & 195.42 & & 381.28 & 167 & 141.43 \\
Willems et al., 2007 & 484.99 & 75 & 134.35 & & 447.27 & 334 & 131.71 \\
\hline
\end{tabular}

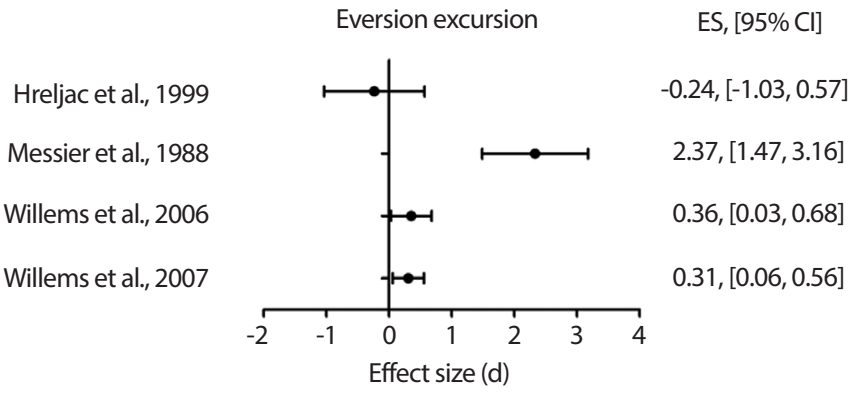

Fig. 4. Eversion excursion Cohen's d effect size and 95\% confidential interval.

the study design was the prospective-cohort design while the other studies [5-9] involved runners for cross-sectional design. Also, type of outcome variables was reported in Table 2 as described in section 2.3. In all studies, the outcome variables were measured between overuse leg injury group and no injury group.

A total of five studies examined rearfoot eversion between overuse injury and control group (Table 3, Fig. 3). The effect size for maximum rearfoot eversion from five studies ranged from -0.34 to 0.67 (All ES and 95\% CI are reported in Fig. 3). Only three of five studies [9,11,12] explain that maximum rearfoot eversion during gait is significantly associated with MTSS, while two studies shows no significant difference in rearfoot eversion between overuse injury and control group. According to Co-
Table 6. Maximum abduction

\begin{tabular}{lcccccccc}
\hline \multirow{2}{*}{ Authors } & \multicolumn{3}{c}{ Treatment } & & \multicolumn{3}{c}{ Control } \\
\cline { 2 - 4 } & $\mathrm{n}$ & $\begin{array}{c}\text { Mean } \\
\left({ }^{\circ}\right)\end{array}$ & SD & & & & $\begin{array}{c}\text { Mean } \\
\left({ }^{\circ}\right)\end{array}$ & SD \\
\hline Franettovich et al., 2010 & -17.7 & 14 & 8.5 & & -22.5 & 14 & 6.9 \\
Milner et al., 2010 & -9.4 & 30 & 5.8 & & -6.7 & 30 & 5.6 \\
Willems et al., 2007 & 12.75 & 75 & 6.31 & & 12.77 & 334 & 6.97 \\
\hline
\end{tabular}

Table 7. Abduction excursion

\begin{tabular}{lccccccccc}
\hline & \multicolumn{3}{c}{ Treatment } & & \multicolumn{3}{c}{ Control } \\
\cline { 2 - 4 } Authors & $\mathrm{n}$ & $\begin{array}{c}\text { Mean } \\
\left({ }^{\circ}\right)\end{array}$ & SD & & & & $\begin{array}{c}\text { Mean } \\
\left({ }^{\circ}\right)\end{array}$ & SD \\
\hline Franettovich et al., 2010 & 27.6 & 14 & 4.5 & & 27.8 & 14 & 6.2 \\
Willems et al., 2006 & 12.92 & 46 & 4.88 & & 11.43 & 167 & 4.02 \\
Willems et al., 2007 & 11.68 & 75 & 4.62 & & 10.55 & 334 & 3.35 \\
\hline
\end{tabular}

Table 8. Maximum abduction velocity

\begin{tabular}{|c|c|c|c|c|c|c|}
\hline \multirow[b]{2}{*}{ Authors } & \multicolumn{3}{|c|}{ Treatment } & \multicolumn{3}{|c|}{ Control } \\
\hline & $\mathrm{n}$ & $\begin{array}{c}\text { Mean } \\
(\% / s)\end{array}$ & SD & $\mathrm{n}$ & $\begin{array}{c}\text { Mean } \\
(\% / s)\end{array}$ & SD \\
\hline Willems et al., 2006 & 435.01 & 46 & 173.52 & 353.66 & 167 & 119.17 \\
\hline Willems et al., 2007 & 337.58 & 75 & 123.52 & 313.76 & 334 & 98.39 \\
\hline
\end{tabular}

Maximum eversion velocity

$\mathrm{ES},[95 \% \mathrm{Cl}]$

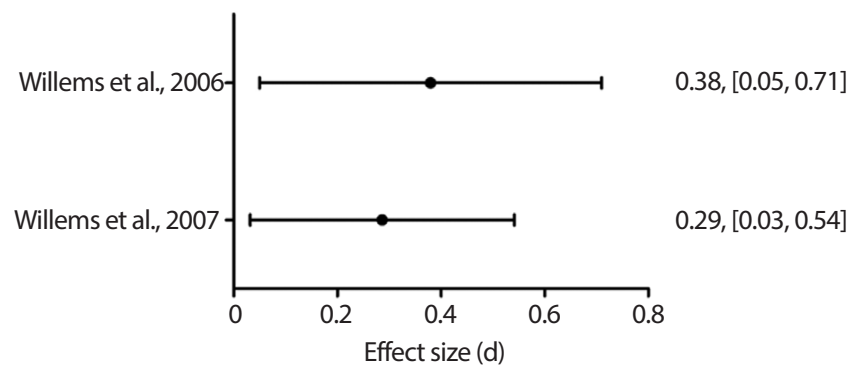

Fig. 5. Maximum eversion velocity Cohen's d effect size and 95\% confidential interval.

hen's d index, all studies [9,11,12] showed medium effect with $0.64,0.67$, and 0.37 , respectively $(0.2 \leq d<0.8)$.

A total of four studies examined rearfoot eversion excursion during gait between overuse injury and control group (Table 4, Fig. 4). The effect size for eversion excursion from 4 studies ranged from -0.24 to 2.37 (All ES and 95\% CI are reported in Fig. 4). Only one of these studies found no significant association with MTSS as a risk factor, while three studies $[7,11,12]$ explain that rearfoot eversion excursion during gait is significantly associated with MTSS as highlighted on ES and 95\% CI. Both studies by Willems et al. [11,12] showed medium effect with 0.31 


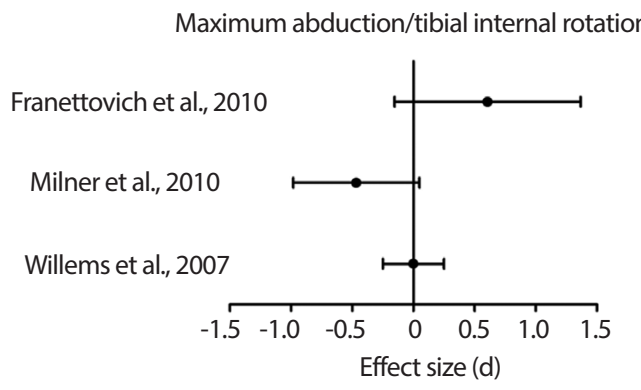

Fig. 6. Maximum abduction / tibial internal rotation Cohen's d effect size and $95 \%$ confidential interval.

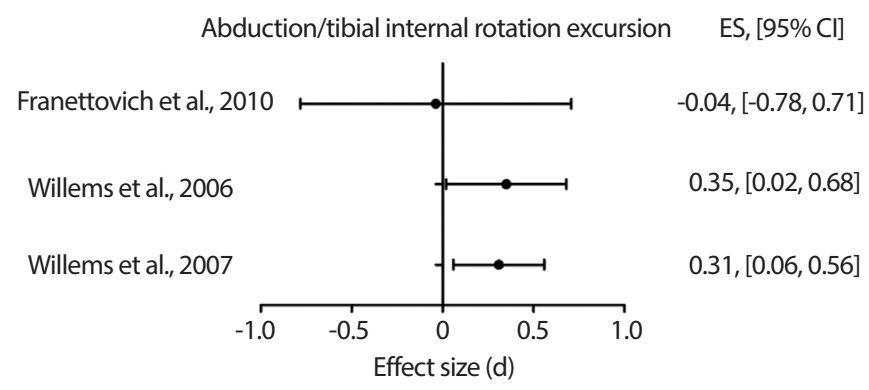

Fig. 7. Abduction/tibial internal rotation excursion Cohen's d effect size and $95 \%$ confidential interval.

and 0.36 , respectively $(0.2 \leq d<0.8)$, while Messier et al., has ES of 2.37 represents a large effect $(d \geq 0.8)$.

There were only two studies reported maximum eversion velocity with effect size 0.29 (95\% CI: 0.03, 0.54) and 0.38 (95\% CI: 0.05, 0.71) from all of 2 studies show statistically significance (Table 5, Fig. 5). Both ES of Willems et al. [11,12] represent medium effect based on Cohen's index $(0.2 \leq d<0.8)$.

Three studies examined foot abduction/tibia internal rotation between overuse injury and control group (Table 6, Fig. 6). In this figure, none of 3 studies statistically significant with the effect sizes were ranged from 0.00 to 0.62 (All ES and 95\% CI are reported in Fig. 6).

Three studies examined foot abduction excursion (Table 7, Fig. 7). The effect size for foot abduction excursion from 3 studies ranged from - 0.04 to 0.35 but two of 3 studies [11,12] statistically significant with ES of 0.35 (95\% CI: 0.02, 0.68) and 0.31 (95\% CI: 0.06, 0.56), respectively, while one study shows no association as a risk factor of MTSS (All ES and 95\% CI are reported in Fig. 7). Two studies by Willems et al. [11,12] represent medium effect with 0.35 and 0.31 , respectively $(0.2 \leq d<0.8)$.

There were only two studies reported maximum foot abduction velocity and both studies [11,12] statistically significant with ES of 0.23 (95\% CI: -0.02, 0.48) and 0.61 (95\% CI: 0.28, 0.94), respectively (Table 8, Fig. 8). The ES of 0.61 and 0.23 from Willems et al. [11,12] represent me-
Maximum abduction/tibial internal rotation velocity $\quad \mathrm{ES}$, [95\% Cl]

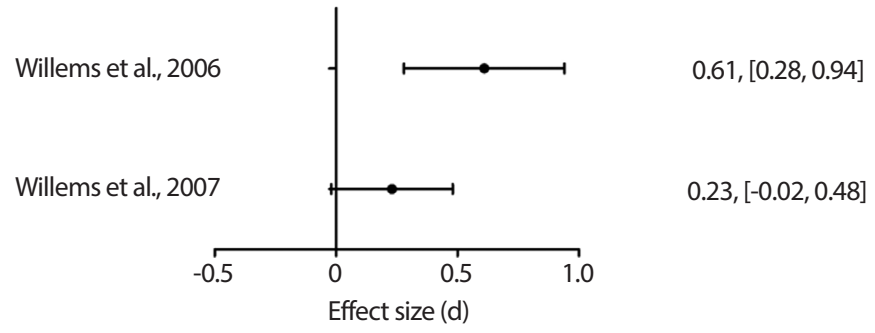

Fig. 8. Maximum abduction velocity Cohen's d effect size and $95 \%$ confidential interval.

dium effect $(0.2 \leq d<0.8$, Table 8 , Fig. 8$)$.

\section{DISCUSSION}

The finding from this review paper confirmed that rearfoot and tibial motion are associated with MTSS during gait. Therefore, we accept the hypothesis; individuals with overuse injuries might have a more subtalar joint motion in the frontal (rearfoot motion) and transverse planes (tibial motion) based on the deviation of subtalar joint.

With the rearfoot kinematic variables from the literature, we found that most selected studies result in consistent greater rearfoot kinematic values in overuse leg injuries than healthy leg. However, one of the selected studies had a bias in the result because the rearfoot motion in healthy group is larger than MTSS group [6]. Most studies found that rearfoot motion in individuals with MTSS is greater than healthy individuals $[7-9,11,12]$. Therefore, the rearfoot eversion from the study by Hreljac et al. is located on opposite side to the other studies in Fig. 3 and 4. Based on these findings, we are able to strongly suggest that future research to develop a clinical prevention/treatment for restricting rearfoot motion is warranted.

On the other hand, we found that controversial results with the tibial kinematic variables from the same literature. Maximum foot abduction/ tibial internal rotation is not associated with overuse leg injuries during gait. Even though two studies $[11,12]$ showed no significance in maximum foot abduction, they showed that foot abduction excursion and foot abduction velocity are associated with overuse leg injuries during gait. Only study by Franettovich et al. [5] showed consistent negative results in both maximum foot abduction and foot abduction excursion. We were not able to make recommendations on the tibial motion due to the insufficient evidence but we suggest that more future researches need to be conducted to justify a clinical prevention/treatment guideline. 
This review paper used the PEDro score, represents the quality of selected studies. The mean of PEDro scores is 4.3 out of 10 , is not a high quality. As allocation conceal and blinding of selected studies was reported in table 1, no study concealed allocation and blind the examiner. Because of the nature of injury pathology and methodology in selected studies, it is hard to perform a randomized control trial (RCT) study design. No RCT designed studies in literature result in low PEDro scores. Another limitation was sample size of individual selected study. There was only two studies reported power analysis for sample size. This could affect on the power of individual study.

The strength of recommendation (SORT) level is graded a 2A. Based on this level of evidence grade, this review paper suggests that restricting rearfoot motion (eversion) can prevent and treat overuse leg injuries. Restricting tibial motion (internal rotation) may affect on prevention and treatment for overuse leg injuries but it seems the effectiveness of restriction on tibial motion is less than rearfoot motion.

\section{CONCLUSION}

This review paper confirms that the kinematic characteristics of MTSS during gait. The rearfoot motion (eversion) associates with overuse leg injuries more than tibial motion (internal rotation). Based on this finding, the clinicians can consider rearfoot motion (eversion) to develop prevention and treatment program for overuse leg injuries including exercise-related leg pain, MTSS, shin splints, and/or tibial stress fracture. In addition, it is recommended that researchers need to perform RCT design to increase quality of evidence for future researches.

\section{CONFLICT OF INTEREST}

No potential conflict of interest relevant to this article was reported.

\section{AUTHOR CONTRIBUTION}

Conceptualization: HP Jun, Data curation: HP Jun, Formal analysis: HP Jun, E Chang, Methodology: HP Jun, E Chang, Project administration: HP Jun, Visualization: HP Jun, E Chang, Writing - original draft: HP Jun, E Chang, Writing-review \& editing: HP Jun, E Chang.

\section{ORCID}

$\begin{array}{ll}\text { Hyung-Pil Jun } & \text { https://orcid.org/0000-0001-7772-8276 } \\ \text { Eunwook Chang } & \text { https://orcid.org/0000-0001-5876-9275 }\end{array}$

\section{REFERENCES}

1. Yates B, White S. The incidence and risk factors in the development of medial tibial stress syndrome among naval recruits. Am J Sports Med. 2004;32(3):772-80

2. Taunton JE, Ryan MB, Clement DB, McKenzie DC, Lloyd-Smith DR et al. retrospective case-control analsis of 2002 running injuries. Br J Sports Med. 2002;36:95-101.

3. Taunton JE, Ryan MB, Clement DB, McKenzie DC, Lloyd-Smith DR et al. A prospective study of running injuries: the Vacouver Sun Run “in training” clinics. Br J Sports Med. 2003;37:239-44.

4. Moen MH, Tol JL, Weir A, Steunebrink M, De Winter TC. Medial tibial stress syndrome. Sports Med. 2009;39(7):523-46.

5. Franettovich M, Chapman AR, Blanch P, Vicenzino B. Altered neuromuscular control in individuals with exercise-related leg pain. Med Sci Sport Exer. 2010;42(3):546-55.

6. Hreljac A, Marshall RN, Hume PA. Evaluation of lower extremity overuse injury potential in runners. Med Sci Sport Exer. 2000;32(9): 1635-41.

7. Messier SP, Pittala KA. Etiologic factors associated with selected running injuries. Med Sci Sport Exer. 1988;20(5):501-5.

8. Milner CE, Hamill J, Davis IS. Distinct hip and rearfoot kinematics in female runners with a history of tibial stress fracture. J Orthop Sport Phys. 2010;40(2):59-66.

9. Pohl MB, Mullineaux DR, Milner CE, Hamill J, Davis IS. Biomechanical predictors of retrospective tibial stress fractures in runners. J Biomech. 2008;41(6):1160-5.

10. Sommer HM, Vallentyne SW. Effect of foot posture on the incidence of medial tibial stress syndrome. Med Sci Sport Exer. 1995;27(6):8004 .

11. Willems T, Witvrouw E, De C. Gait-related risk factors for exercise-related lower-leg pain during shod running. Med Sci Sport Exer. 2007; 39(2):330-9.

12. Willems TM, De Clercq D, Delbaere K, Vanderstraeten G, De Cock A et al. A prospective study of gait related risk factors for exercise-related lower leg pain. Gait Posture. 2006;23(1):91-8. 
13. Bennett J, Reinking M, Pluemer B, Pentel A, Seaton M et al. Factors contributing to the development of medial tibial stress syndrome in high school runners. J Orthop Sport Phys. 2001;31(9):504-10.

14. Raissi GRD, Cherati ADS, Mansoori KD, Razi MD. The relationship between lower extremity alignment and Medial Tibial Stress Syndrome among non-professional athletes. Sports Med Arthrosc Rehabil Ther Technol. 2009;1(1):1-8.

15. Reinking MF. Exercise-related leg pain in female collegiate athletes: the influence of intrinsic and extrinsic factors. Am J Sport Med. 2006; 34(9):1500-7.
16. Smith R, Rattanaprasert U, O'Dwyer N. Coordination of the ankle joint complex during walking. Hum Movement Sci. 2001;20(4-5):44760.

17. de Asla RJ, Wan L, Rubash HE, Li G. Six DOF in vivo kinematics of the ankle joint complex: Application of a combined dual-orthogonal fluoroscopic and magnetic resonance imaging technique. J Orthop Res. 2006;24(5):1019-27.

18. Wesley RA. Investigation of Biomechanical Risk Factors of Medial Tibial Stress Syndrome through Finite Element Analysis: North Carolina Agricultural and Technical State University; 2015. 